\title{
Row-column factorial designs with multiple levels
}

\author{
Fahim Rahim and Nicholas J. Cavenagh \\ Department of Mathematics and Statistics, \\ University of Waikato, Private Bag 3105, \\ Waikato Mail Centre 3240, New Zealand
}

January 18, 2021

\begin{abstract}
An $m \times n$ row-column factorial design is an arrangement of the elements of a factorial design into a rectangular array. Such an array is used in experimental design, where the rows and columns can act as blocking factors. If for each row/column and vector position, each element has the same regularity, then all main effects can be estimated without confounding by the row and column blocking factors.

Formally, for any integer $q$, let $[q]=\{0,1, \ldots, q-1\}$. The $q^{k}$ (full) factorial design with replication $\alpha$ is the multi-set consisting of $\alpha$ occurrences of each element of $[q]^{k}$; we denote this by $\alpha \times[q]^{k}$. A regular $m \times n$ row-column factorial design is an arrangement of the the elements of $\alpha \times[q]^{k}$ into an $m \times n$ array (which we say is of type $I_{k}(m, n ; q)$ ) such that for each row (column) and fixed vector position $i \in[q]$, each element of $[q]$ occurs $n / q$ times (respectively, $m / q$ times). Let $m \leq n$. We show that an array of type $I_{k}(m, n ; q)$ exists if and only if (a) $q \mid m$ and $q \mid n$; (b) $q^{k} \mid m n$; (c) $(k, q, m, n) \neq(2,6,6,6)$ and (d) if $(k, q, m)=(2,2,2)$ then 4 divides $n$. This extends the work of Godolphin (2019), who showed the above is true for the case $q=2$ when $m$ and $n$ are powers of 2 .

In the case $k=2$, the above implies necessary and sufficient conditions for the existence of a pair of mutually orthogonal frequency rectangles (or $F$-rectangles) whenever each symbol occurs the same number of times in a given row or column.
\end{abstract}

Keywords: Row-column factorial design, blocking factor, double confounding, frequency square or $F$-square, frequency rectangle or $F$-rectangle, MOFS.

\section{Introduction}

For any integer $q$, let $[q]=\{0,1, \ldots, q-1\}$. Consider the following example of an experimental design from [8]. Suppose we wish to study the effects of four drugs on calves, where there are four breeds, four age groups and two levels for dosage for each type of drug. We could conduct 16 experiments based on the following row-column factorial design: 


$\begin{array}{llll}1111 & 0100 & 0010 & 1001 \\ 0001 & 1010 & 1100 & 0111 \\ 1000 & 0011 & 0101 & 1110 \\ 0110 & 1101 & 1011 & 0000\end{array}$

Table 1: A regular row-column factorial design of type $I_{4}(4,4 ; 2)$.

Here the rows and columns correspond to age groups and breeds, respectively, of calves; with the binary vector in a cell indicating the dosage of each of the four drugs as one of two levels.

In the above the 16 vectors from $[2]^{4}$ are arranged in a $4 \times 4$ array, in such a way that for each row (column) and $i \in[4]$, the entries 0 and 1 each appear twice in position $i$ of a vector in that row (respectively, column). These properties of regularity in the experimental design allows unbiased estimation of the effects of each drug, together with the effects of breed and age group (which can be thought of as blocking factors), without any confounding between these main effects.

Formally, the $q^{k}$ (full) factorial design with replication $\alpha$ is the multi-set consisting of $\alpha$ occurrences of each element of $[q]^{k}$; we denote this by $\alpha \times[q]^{k}$. An $m \times n$ row-column factorial design $q^{k}$ is any arrangement of the elements of $\alpha \times q^{k}$ into an $m \times n$ array. Necessarily, $q^{k}$ must divide $m n$. Without loss of generality, we always assume $m \leq n$. We call such a design regular if for each row (column) and $i \in[q]$, each element of $[q]$ occurs $n / q$ times (respectively, $m / q$ times). Furthermore we denote the type of such an array to be $I_{k}(m, n ; q)$, where regularity is always assumed to hold. Observe that regularity implies that $q$ divides both $m$ and $n$. The above example is thus a regular $4 \times 4$ row-column factorial design $2^{4}$, or equivalently an array of type $I_{4}(4,4 ; 2)$. Note that an array of type $I_{2}(n, n ; n)$ is equivalent to a pair of orthogonal Latin squares of order $n$.

We first review the impact of row-column factorial designs within experimental design literature. A blocking factor can be thought of as a partition of the blocks of a design, typically an equipartition (all subsets in the partition have equal size) with further properties of regularity to minimize estimation bias within the design structure. Blocking factors for factorial designs have been well-studied ([1, [2, [9], 10, [16]). However, as mentioned in [13], having two forms of blocking for a factorial design is less well-studied.

Within experimental design a row-column design can refer to a variety of combinatorial designs, all with the property of being arranged in a rectangular array, where the rows and columns are typically (but not always) blocking factors. This is sometimes referred to as double confounding [13]. To ensure that certain effects can be estimated without bias being introduced by the design, regularity conditions are imposed. For example, in a Latin square each symbol occurs once per row and once per column. While some examples of row-column factorial designs are given in 25, the earliest known example in the literature of a regular row-column factorial design is given in [21], where an array of type $I_{5}(8,8 ; 2)$ is featured. In practice non-regular row-column factorial designs are also sometimes of use. In [24], a non-regular row-column factorial design is given which was used by the CSIRO Division of Forestry for a glasshouse experiment. Here the physical distance to the edge of the glasshouse is an important factor. 
A quasi-Latin square is an $n \times n$ array such that for some $k>n$ which divides $n^{2}$, each entry from $[k]$ occurs $n^{2} / k$ times in the array, with no entry occurring more than once per row or column. Some of the literature on quasi-Latin squares features row-column factorial designs [3]. Here if we consider the vectors as the entries, a row-column factorial design can be thought of as a quasi-Latin square if no vector occurs more than once in a row or column (necessarily, $m, n<2^{k}$ ). John and Lewis 15 describe a technique to cyclically generate some row-column factorial designs. Examples and methods to construct rowcolumn factorial designs are also given in [6], [7] and [5]. Wang [23] constructs $I_{k}\left(2^{M}, 2^{N} ; 2\right)$ whenever $k=M+N$. A variation of row-column factorial designs is considered by [8]: a generalized confounded row-column design can be thought of as a factorial design arranged into a rectangular array where each cell contains a constant number of vectors.

Row-column factorial designs with two levels (that is, $q=2$ ) are studied in [13]. As well as the result in Theorem 1 below, designs are also constructed to estimate paired interactions without confounding by row and column blocking factors.

Theorem 1. ([13]) Let $1 \leq M \leq N$. A regular $2^{M} \times 2^{N}$ row-column factorial design $2^{k}$ exists if and only if $k \leq M+N$ and $(k, M, N) \neq(2,1,1)$.

Our main result is generalizing the above to arrays of arbitrary dimensions and number of levels (that is, arbitrary $m, n, q$ and $k$ ).

Theorem 2. Let $m \leq n$. There exists a regular $m \times n$ row-column factorial design $q^{k}$ if and only if $q$ divides $m, q$ divides $n, q^{k}$ divides $m n$ unless:

i. $k=q=m=2$ and $n \equiv 2(\bmod 4)$.

ii. $k=2$ and $q=m=n=6$.

We also show that the above exceptions are genuine exceptions; i.e. the above theorem describes necessary and sufficient existence conditions.

We next describe the connection between regular row-column factorial designs and frequency rectangles. Given two vectors $\mathbf{v}=\left(v_{0}, v_{1}, \ldots, v_{k-1}\right)$ and $\mathbf{w}=\left(w_{0}, w_{1}, \ldots, w_{\ell-1}\right)$, we define $\mathbf{v} \oplus \mathbf{w}$ to be the concatenation of $\mathbf{v}$ and $\mathbf{w}$, that is:

$$
\mathbf{v} \oplus \mathbf{w}:=\left(v_{0}, v_{1}, \ldots, v_{k-1}, w_{0}, w_{1}, \ldots, w_{\ell-1}\right) .
$$

Next, let $A=\left[a_{i j}\right]$ and $B=\left[b_{i j}\right]$ be matrices of the same dimensions, with each entry of $A$ is a vector of dimension $k$ and each entry of $B$ is a vector of dimension $\ell$. Then we define $C=A \oplus B$ to be the matrix given by $C=\left[c_{i j}:=a_{i j} \oplus b_{i j}\right]$.

Now, an array of type $I_{k}(m, n ; q)$ can be written in the form $F_{0} \oplus F_{1} \oplus \cdots \oplus F_{k-1}$, where each entry of each $F_{i}, i \in[k]$, has dimension 1 . Since regularity is assumed, each element of $[q]$ occurs precisely $n / q$ times per row and $m / q$ times per column, for each of the arrays $F_{i}, i \in[k]$. These arrays are thus frequency rectangles.

Formally, a frequency rectangle (sometimes known as an $F$-rectangle) of type $F R(m, n ; q)$ is an $m \times n$ array such that each element of $[q]$ occurs $m / q$ times per row and $n / q$ times per column. Thus, we may write any array of type $I_{k}(m, n ; q)$ as $F_{0} \oplus F_{1} \oplus \cdots \oplus F_{k-1}$, where for each $i \in[k], F_{i}$ is a frequency rectangle of type $F R(m, n ; q)$. We note here that frequency rectangles in the literature (most often frequency squares or $F$-squares when 
$m=n)$ may have different row/column frequencies for distinct symbols. In this paper we restrict ourselves to the regular case.

Two frequency rectangles of type $F R(m, n ; q)$ are orthogonal if, when superimposed, each ordered pair from $[q] \times[q]$ occurs exactly $m n / q^{2}$ times in the array. A set of pairwise orthogonal frequency rectangles are called mutually orthogonal rectangles. These have mostly been studied in the case $m=n$, where such structures are called Mutually Orthogonal Frequency Squares or MOFS).

The existence problem for pairs of MOFS has been completely classified; the following theorem is a special case of [17, p. 67]. The exceptions are precisely the two orders for which pairs of MOLS which do not exist, as known by Euler.

Theorem 3. There exists a pair of MOFS of type $F(n, n ; q)$ if and only if $(n, q) \notin$ $\{(2,1),(6,1)\}$.

Hedayat, Raghavarao, et al. 14] showed that if a set of $k$ MOFS of type $F R(n, n ; q)$ exists then $k \leq(n-1)^{2} /(q-1)$. When $k$ meets this upper bound such a set is called complete. Complete sets of MOFS exist when $q=2$ and if there exists a Hadamard matrix of order $n$ [12; otherwise they are only known to exist when $n$ is a prime power [18, 19, 20, 22]. A complete sets of MOFS does not exist when $q=2$ and $n \equiv 2(\bmod 4)$ [4.

Note that while an array of type $I_{k}(m, n ; q)$ yields a set of $k$ mutually orthogonal frequency rectangles, the converse is not always true for $k \geq 3$, as seen below. Here we see a set of three MOFS (overlapped) which is not a row-column factorial design.

$\begin{array}{llllll}000 & 111 & 000 & 101 & 011 & 110 \\ 111 & 000 & 000 & 011 & 110 & 101 \\ 000 & 000 & 111 & 110 & 101 & 011 \\ 101 & 011 & 110 & 010 & 100 & 001 \\ 011 & 110 & 101 & 100 & 001 & 010 \\ 110 & 101 & 011 & 001 & 010 & 100\end{array}$

Three MOFS of type $F(6,6 ; 3)$

However, if $k=2$ an $I_{k}(m, n ; q)$ is equivalent to a pair of mutually orthogonal frequency rectangles of type $F(m, n ; q)$.

Theorem 4. [11 Let $q$ divide $m$ and $n$. If $q \notin\{2,6\}$ or at least one of $n / q, m / q$ is even, there exists a pair of mutually orthogonal frequency rectangles of type $F(m, n ; q)$ (equivalently, an array of type $I_{2}(m, n ; q)$ ).

In Section 4, we generalize the previous theorem to find necessary and sufficient conditions for the existence of an array of type $I_{2}(m, n ; q)$. We prove the remaining cases of Theorem 2 in Section 5, using the recursive constructions from Section 2 and the finite field constructions from Section 3. 


\section{Recursive constructions}

In this section we discuss ways in which row-column factorial designs can be built recursively. We begin with a straightforward lemma.

Lemma 1. If there exists arrays of type $I_{k}(m, n ; q)$ and $I_{k}\left(m^{\prime}, n ; q\right)$ there exists an array of type $I_{k}(m+m, n ; q)$. If there exists arrays of type $I_{k}(m, n ; q)$ and $I_{k}\left(m, n^{\prime} ; q\right)$ there exists an array of type $I_{k}\left(m, n+n^{\prime} ; q\right)$.

Next we consider a type of Kronecker Product. Let $A=\left[a_{i j}\right]$ and $B=\left[b_{i j}\right]$ be two arrays of sizes $m \times n$ and $u \times v$, respectively. The Kronecker product, $A \otimes B$, of $A$ and $B$ is an $m u \times n v$ array defined by:

$$
A \otimes B=\left(\begin{array}{ccc}
a_{11} B & \ldots & a_{1 n} B \\
\vdots & & \vdots \\
a_{m 1} B & \ldots & a_{m n} B
\end{array}\right)
$$

where $a_{l k} B$ is a $u \times v$ array with the entry $(i, j)$ given by $\left(a_{l k}, b_{i j}\right)$.

It is easy to see that if $F$ and $F^{\prime}$ are two frequency rectangles of type $F R(m, n ; q)$ and $F R\left(m^{\prime}, n^{\prime} ; q^{\prime}\right)$ respectively, then their Kronecker product $F \otimes F^{\prime}$ is a frequency rectangle of type $F R\left(m m^{\prime}, n n^{\prime} ; q q^{\prime}\right)$, where the entries of $[q] \times\left[q^{\prime}\right]$ are mapped to $\left[q q^{\prime}\right]$ by some bijection $f$. In this fashion, let $D=F_{0} \oplus F_{1} \oplus \cdots \oplus F_{k-1}$ and $D^{\prime}=F_{0}^{\prime} \oplus F_{1}^{\prime} \oplus \cdots \oplus F_{k-1}^{\prime}$ be two row-column factorial designs of types $I_{k}(m, n ; q)$ and $I_{k}\left(m^{\prime}, n^{\prime} ; q^{\prime}\right)$, respectively, where $F_{i}$ and $F_{i}^{\prime}$ are frequency rectangles for each $i \in[k]$. Then we define $D \otimes D^{\prime}$ to be the array given by $\left(F_{0} \otimes F_{0}^{\prime}\right) \oplus\left(F_{1} \otimes F_{1}^{\prime}\right) \oplus \cdots \oplus\left(F_{k-1} \otimes F_{k-1}^{\prime}\right)$.

Lemma 2. If $D$ and $D^{\prime}$ are arrays of type $I_{k}(m, n ; q)$ and $I_{k}\left(m^{\prime}, n^{\prime} ; q^{\prime}\right)$, respectively, then $D \otimes D^{\prime}$, as defined above, is an array of type $I_{k}\left(m m^{\prime}, n n^{\prime} ; q q^{\prime}\right)$.

Proof. It suffices to show that the entries of the cells of $D \otimes D^{\prime}$ form a factorial design. Let $D=F_{0} \oplus F_{1} \oplus \cdots \oplus F_{k-1}$ and $D^{\prime}=F_{0}^{\prime} \oplus F_{1}^{\prime} \oplus \cdots \oplus F_{k-1}^{\prime}$ as above.

Consider any $\left(\alpha_{0}, \alpha_{1}, \ldots \alpha_{k-1}\right) \in\left[q q^{\prime}\right]$ in a cell of $D \otimes D^{\prime}$. Then for each $i \in[k], \alpha_{i}=$ $f\left(a_{i}, b_{i}\right)$, for some $a_{i}$ and $b_{i}$ belong to the symbol sets of $F_{i}$ and $F_{i}^{\prime}$ respectively. Since $D$ is of type $I_{k}(m, n ; q)$, there are precisely $m n / q^{k}$ cells containing $a_{i}$ in $F_{i}$ for each $i \in[k]$. Similarly, there are exactly $m^{\prime} n^{\prime} /\left(q^{\prime}\right)^{k}$ cells containing $b_{i}$ in $F_{i}^{\prime}$ for each $i \in[k]$. From the definition of the Kronecker product, the sequence $\left(\alpha_{0}, \alpha_{1}, \ldots, \alpha_{k-1}\right)$ appears exactly $m m^{\prime} n n^{\prime} /\left(q q^{\prime}\right)^{k}$ times in $D \otimes D^{\prime}$.

Example 1. Consider the two arrays $D$ and $D^{\prime}$ of type $I_{3}(4,2 ; 2)$ and $I_{3}(3,9 ; 3)$ respectively.

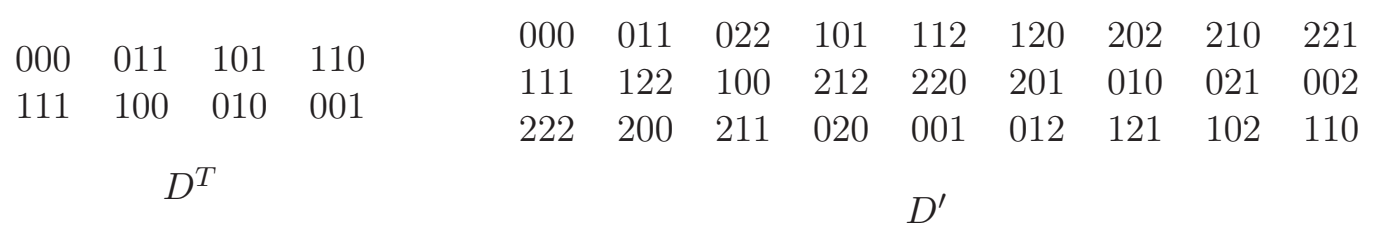

Then by taking the product $D \otimes D^{\prime}$ and using the bijection $(a, b) \mapsto 3 a+b$ to transform the symbol set, we get an array of type $I_{3}(12,18 ; 6)$ : 


$\begin{array}{llllllllllllllllll}000 & 011 & 022 & 101 & 112 & 120 & 202 & 210 & 221 & 333 & 344 & 355 & 434 & 445 & 453 & 535 & 543 & 554 \\ 111 & 122 & 100 & 212 & 220 & 201 & 010 & 021 & 002 & 444 & 455 & 433 & 545 & 553 & 534 & 343 & 354 & 335 \\ 222 & 200 & 211 & 020 & 001 & 012 & 121 & 102 & 110 & 555 & 533 & 544 & 353 & 334 & 345 & 454 & 435 & 443 \\ 033 & 044 & 055 & 134 & 145 & 153 & 235 & 243 & 254 & 300 & 311 & 322 & 401 & 412 & 420 & 502 & 510 & 521 \\ 144 & 155 & 133 & 245 & 253 & 234 & 043 & 054 & 035 & 411 & 422 & 400 & 512 & 520 & 501 & 310 & 321 & 302 \\ 255 & 233 & 244 & 053 & 034 & 045 & 154 & 135 & 143 & 522 & 500 & 511 & 320 & 301 & 312 & 421 & 402 & 410 \\ 303 & 314 & 325 & 404 & 415 & 423 & 505 & 513 & 524 & 030 & 041 & 052 & 131 & 142 & 150 & 232 & 240 & 251 \\ 414 & 425 & 403 & 515 & 523 & 504 & 313 & 324 & 305 & 141 & 152 & 130 & 242 & 250 & 231 & 040 & 051 & 032 \\ 525 & 503 & 514 & 323 & 304 & 315 & 424 & 405 & 413 & 252 & 230 & 241 & 050 & 031 & 042 & 151 & 132 & 140 \\ 330 & 341 & 352 & 431 & 442 & 450 & 532 & 540 & 551 & 003 & 014 & 025 & 104 & 115 & 123 & 205 & 213 & 224 \\ 441 & 452 & 430 & 542 & 550 & 531 & 340 & 351 & 332 & 114 & 125 & 103 & 215 & 223 & 204 & 013 & 024 & 005 \\ 552 & 530 & 541 & 350 & 331 & 342 & 451 & 432 & 440 & 225 & 203 & 214 & 023 & 004 & 015 & 124 & 105 & 113\end{array}$

An array of type $I_{3}(12,18 ; 6)$

Corollary 1. If there exist $r$ arrays of types $I_{k}\left(m_{i}, n_{i} ; q_{i}\right)$, where $i \in[r]$, then there exist an array of type $I_{k}\left(\prod_{i=0}^{r-1} m_{i}, \prod_{i=0}^{r-1} n_{i} ; \prod_{i=0}^{r-1} q_{i}\right)$.

Trivially there exists an array of type $I_{k}(m, n ; 1)$ for any integers $k, m, n$. The following corollary is then immediate.

Corollary 2. If there exists an array of type $I_{k}(m, n ; q)$, then there exists an array of type $I_{k}\left(m m^{\prime}, n n^{\prime} ; q\right)$ for any integers $m^{\prime}, n^{\prime} \geq 1$.

\section{Prime power constructions}

In this section, we construct row-column factorial designs via finite fields of prime power order $q$. It is implicitly understood that field elements are relabelled to elements of $[q]$ as a final step in construction.

Lemma 3. Let $M, N \geq 1$ and $q \geq 2$, with $(M, N, q) \neq(1,1,2)$. Then there exists a linearly independent set of $M+N$ polynomials:

$$
f_{r}\left(x_{0}, \ldots, x_{M+N-1}\right)=a_{r, 0} x_{0}+a_{r, 1} x_{1}+\cdots+a_{r, M+N-1} x_{M+N-1} ; r \in[M+N]
$$

over the field $\mathbb{F}_{q}$ which satisfy the following two conditions for each $r \in[M+N]$ :

(i) $\left(a_{r, 0}, \ldots, a_{r, M-1}\right) \neq(0, \ldots, 0)$;

(ii) $\left(a_{r, M}, \ldots, a_{r, M+N-1}\right) \neq(0, \ldots, 0)$.

Proof. We split the proof into cases.

Case I: When $M=N=1$ and $q>2$.

In this case we can take the following two polynomials:

$$
\begin{aligned}
& f_{0}\left(x_{0}, x_{1}\right)=x_{0}+x_{1} \\
& f_{1}\left(x_{0}, x_{1}\right)=x_{0}+\alpha x_{1},
\end{aligned}
$$


where $\alpha$ is a non-zero element in $\mathbb{F}_{q}$ other than identity.

Case II (a): When $N \geq 2$ and $q$ is a power of 2 .

Consider the identity matrix $I_{M+N}$ of order $M+N$. By performing the following two row operations sequentially:

$$
\begin{aligned}
& R_{0}+R_{s} \rightarrow R_{s} \quad \text { for each } s \in\{1,2, \ldots, M+N-1\} \\
& R_{s}+\left(R_{M+N-1}+R_{M+N}\right) \rightarrow R_{s} \quad \text { for each } s \in[M]
\end{aligned}
$$

we get the following matrix:

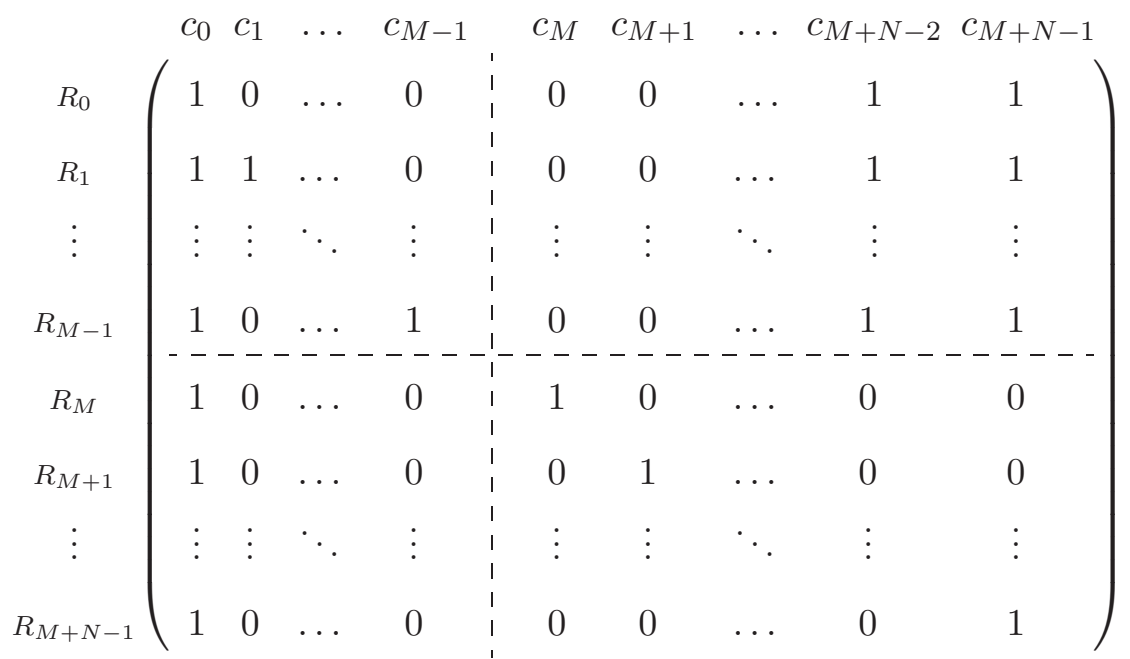

Now corresponding to each row $R_{s}=\left(r_{s, 0}, \ldots, r_{s,(M+N-1)}\right)$ of the above matrix we define a polynomial $f_{s}=r_{s, 0} x_{0}+\cdots+r_{s,(M+N-1) x_{M+N-1}}$ in $\mathbb{F}_{q}$, where, $s \in[M+N]$. Then these polynomials satisfy the conditions (i) and (ii) and are linearly independent.

Case II (b): When $N \geq 2$ and $q$ is not a power of 2 .

In this case again take the identity matrix $I_{M+N}$ and by replacing the second row operation in (11) by $R_{s}+R_{M+N} \rightarrow R_{s}$ for each $s \in[M]$, we get the following matrix:

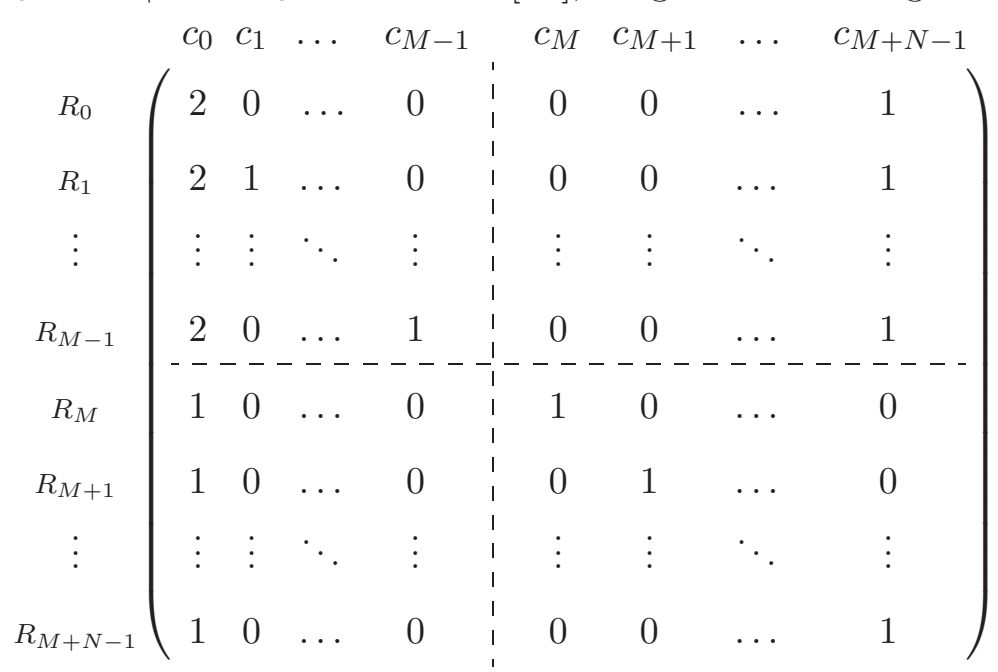

It is easy to see that the corresponding polynomials are linearly independent in $\mathbb{F}_{q}$ and satisfy the conditions (i) and (ii). 
Theorem 5. Let $q \geq 2$ be a prime power. Let $M, N \geq 1$ and $(M, N, q) \neq(1,1,2)$. There exists an array of type $I_{M+N}\left(q^{M}, q^{N} ; q\right)$.

Proof. First we describe a method to construct a frequency rectangle of type $F R\left(q^{M}, q^{N} ; q\right)$ corresponding to polynomial $f_{r}$ for each $r \in[M+N]$, as given by Lemma 3 ,

Label the rows and columns of a $q^{M} \times q^{N}$ array, respectively, by using the set of all $M$-tuples and $N$-tuples over the field $\mathbb{F}_{q}$. Now consider a polynomial

$$
f_{r}\left(x_{0}, \ldots, x_{M+N-1}\right)=a_{r, 0} x_{r, 0}+\cdots+a_{r, M+N-1} x_{r, M+N-1}
$$

over the field $\mathbb{F}_{q}$ that satisfies the conditions given in Lemma 3. We place the element $f\left(b_{0}, \ldots, b_{M-1}, c_{0}, \ldots, c_{N-1}\right)$ in the intersection of row $\left(b_{0}, \ldots, b_{M-1}\right)$ and column $\left(c_{0}, \ldots, c_{N-1}\right)$ of the $q^{M} \times q^{N}$ array.

Now we show that the array obtained in this way is a frequency rectangle of type $F R\left(q^{M}, q^{N} ; q\right)$, that is every element of $\mathbb{F}_{q}$ appears exactly $q^{N-1}$ times in each row and $q^{M-1}$ times in each column. Consider a row which is labelled by $\left(b_{0}, \ldots, b_{M-1}\right)$ and take an element $\alpha \in \mathbb{F}_{q}$. For this row, the equation

$$
f_{r}\left(b_{0}, \ldots, b_{M-1}, x_{M}, \ldots, x_{M+N-1}\right)=\alpha
$$

reduces to the equation

$$
K+a_{r, M} x_{M}+\ldots+a_{r, M+N-1} x_{M+N-1}=\alpha
$$

where $K$ is a constant. Now by axiom (ii) of Lemma 3 there exists $i \in\{M, M+1, \ldots, M+$ $N-1\}$ such that $a_{r, i} \neq 0$. We solve the equation (2) for $x_{i}$ :

$$
x_{i}=\frac{1}{a_{r, i}}\left(\alpha-K-a_{r, M} x_{M}-\cdots-a_{r, i-1} x_{i-1}-a_{r, i+1} x_{i+1}-\cdots-a_{r, M+N-1} x_{M+N-1}\right) .
$$

Since there are $q$ elements in $\mathbb{F}_{q}$ and the $N-1$ variables on the right side of (3) can take any value from $\mathbb{F}_{q}$, the equation (3) has exactly $q^{N-1}$ solutions in $\mathbb{F}_{q}$. This implies that the symbol $\alpha$ appears exactly at $q^{N-1}$ places in the row $\left(b_{0}, \ldots, b_{M-1}\right)$. By a similar argument we can prove that each symbol appears exactly $q^{M-1}$ times in each column. Thus the resulting array is a frequency rectangle of type $F R\left(q^{M}, q^{N} ; q\right)$.

Now to construct an array of type $I_{M+N}\left(q^{M}, q^{N} ; q\right)$. Consider a set of $M+N$ linearly independent polynomials

$$
f_{r}\left(x_{0}, \ldots, x_{M+N-1}\right)=a_{r, 0} x_{0}+\cdots+a_{r, M+N-1} x_{M+N-1} ; \quad r \in[M+N]
$$

over the field $\mathbb{F}_{q}$, such that the coefficients satisfy the conditions (i) and (ii) of Lemma 3 , As above, for each $r \in[M+N]$ we obtain a frequency rectangle $F_{r}$ of type $F R\left(q^{M}, q^{N} ; q\right)$ It remains to show that $F_{0} \oplus F_{1} \oplus \cdots \oplus F_{M+N-1}$ is an array of type $I_{M+N}\left(q^{M}, q^{N} ; q\right)$. To this end, consider any $\left(\alpha_{0}, \alpha_{1}, \ldots, \alpha_{M+N-1}\right) \in\left(\mathbb{F}_{q}\right)^{M+N}$. Since the polynomials above are linearly independent, the system of equations:

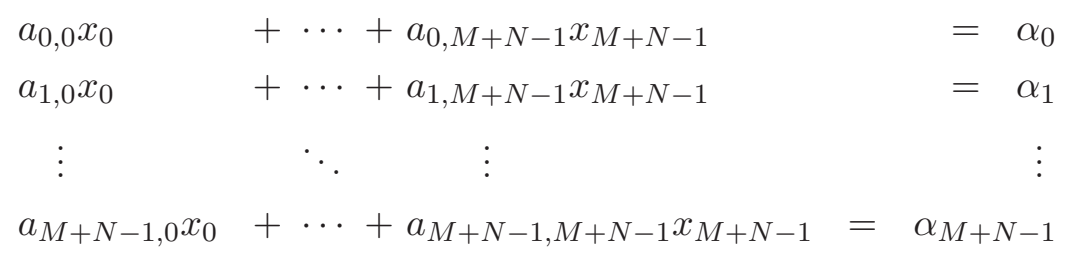


has rank $M+N$ and therefore has a unique solution in $\mathbb{F}_{q}$, which shows that $\left(\alpha_{0}, \alpha_{1}, \ldots, \alpha_{M+N-1}\right)$ appears in exactly one cell of the array constructed.

Corollary 2 implies the following:

Corollary 3. If $(q, M, N) \neq(2,1,1)$, there exist an array of type $I_{M+N}\left(q^{M} b_{1}, q^{N} b_{2} ; q\right)$, for any prime power $q$.

\section{1 "Sudoku" Frequency Rectangles}

In this subsection we take the construction above and take it one step further. Specifically, we show in Theorem 7 that if $q$ divides $b_{1} b_{2}$ and $q$ is a prime power, there exists an array of type $I_{M+N+1}\left(q^{M} b_{1}, q^{N} b_{2} ; q\right)$.

First we describe a Latin square which has a Sudoku-type property with $q=q_{1} q_{2}$ symbols, where $q_{1}$ and $q_{2}$ are positive integers and the symbol set is taken to be $[q]$. That is, such a Latin square can be partitioned into $q_{1} \times q_{2}$ subarrays containing each element of $[q]$.

Theorem 6. Let $q_{1}, q_{2} \geq 1$. Then there exists a Latin square $L\left(q_{1}, q_{2}\right)$ of order $q_{1} q_{2}$ such that for each $i \in\left[q_{1}\right]$ and $j \in\left[q_{2}\right]$, the set of cells

$$
\left\{\left(i^{\prime}, j^{\prime}\right) \mid i \equiv i^{\prime} \quad\left(\bmod q_{1}\right), j \equiv j^{\prime} \quad\left(\bmod q_{2}\right)\right\}
$$

contain each entry from $\left[q_{1} q_{2}\right]$ exactly once.

Proof. Let $q=q_{1} \times q_{2}$, Consider the following array of size $q \times q$ partitioned into $q$ subarrays $S_{0}, S_{1}, \ldots, S_{q-1}$, each of size $q_{1} \times q_{2}$ :

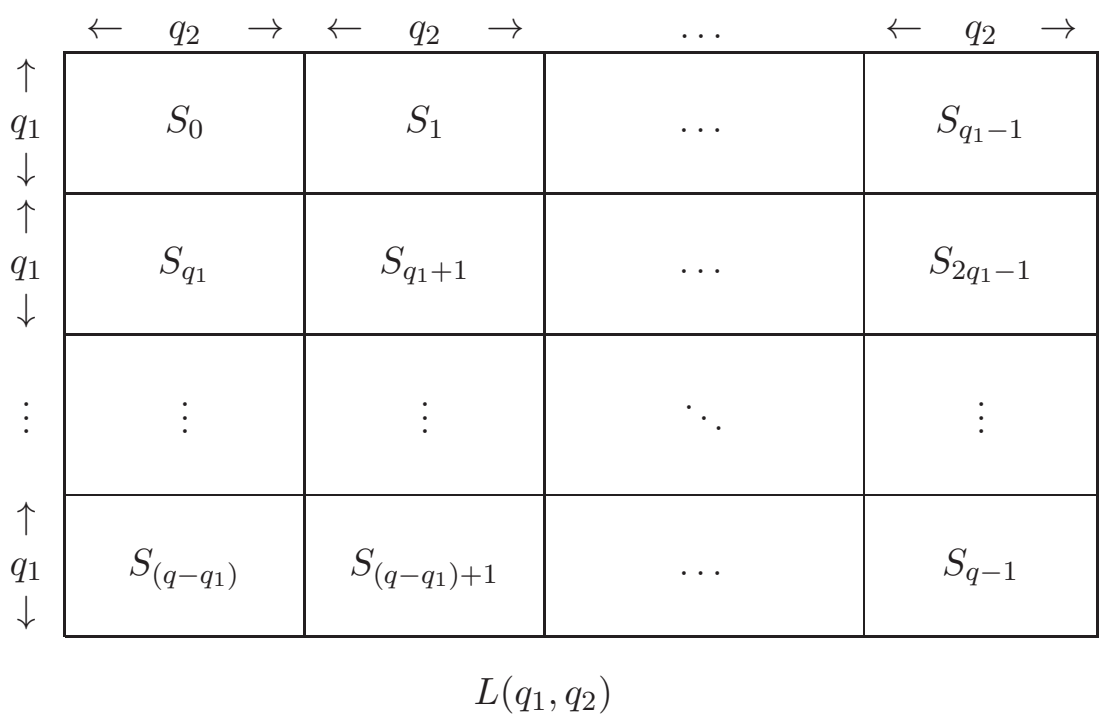

We next describe the structure of $S_{0}$. Arrange the $q$ symbols in the sub array $S_{0}$ in ascending order starting from column 1 . Thus $S_{0}$ has the following form: 


\begin{tabular}{|cccc|}
\hline 0 & $q_{1}$ & $\ldots$ & $\left(q-q_{1}\right)$ \\
1 & $q_{1}+1$ & $\ldots$ & $\left(q-q_{1}\right)+1$ \\
& & & \\
$\vdots$ & $\vdots$ & $\ddots$ & $\vdots$ \\
& & & \\
$q_{1}-1$ & $2 q_{1}-1$ & $\ldots$ & $q-1$ \\
\hline
\end{tabular}

$S_{0}$

Now we define the subarray $S_{i}$ to be the array obtained by adding the symbol $i(\bmod q)$ to each cell of $S_{0}$. Finally arrange these $S_{i}$ 's as described above to obtain $L\left(q_{1}, q_{2}\right)$.

Example 2. We exhibit the previous construction in the case $q=6, q_{1}=2$ and $q_{2}=3$ :

\begin{tabular}{|lll|lll|}
\hline 0 & 2 & 4 & 1 & 3 & 5 \\
1 & 3 & 5 & 2 & 4 & 0 \\
\hline 2 & 4 & 0 & 3 & 5 & 1 \\
3 & 5 & 1 & 4 & 0 & 2 \\
\hline 4 & 0 & 2 & 5 & 1 & 3 \\
5 & 1 & 3 & 0 & 2 & 4 \\
\hline
\end{tabular}

$L(2,3)$

Now, a Latin square of order $q$ is also an array of type $I_{1}(q, q ; q)$. Thus, from Corollary 2. we have the following corollary.

Corollary 4. For any integers $\mu, \lambda, q_{1}, q_{2} \geq 1$, there exists a frequency rectangle of type $F R(q \mu, q \lambda ; q)$ (where $q=q_{1} q_{2}$ ) such that for each $i \in\left[\mu q_{1}\right]$ and $j \in\left[\lambda q_{2}\right]$, the set of cells

$$
\left\{\left(i^{\prime}, j^{\prime}\right) \mid i \equiv i^{\prime} \quad\left(\bmod \mu q_{1}\right), j \equiv j^{\prime} \quad\left(\bmod \lambda q_{2}\right)\right\}
$$

contain each entry from $\left[q_{1} q_{2}\right]$ exactly once.

Example 3. If $L$ is the Latin square $L(2,3)$, then $L \otimes I_{1}(2,3 ; 1)$ yields a frequency rectangle of type $F R(12,18 ; 6)$. The entries in bold show the elements of $[6]$ occurring in cells of the form $(i, j)$ where $i \equiv 1(\bmod 4)$ and $j \equiv 2(\bmod 9)$. 


\begin{tabular}{|lllllllll|lllllllll|}
\hline 0 & 0 & 0 & 2 & 2 & 2 & 4 & 4 & 4 & 1 & 1 & 1 & 3 & 3 & 3 & 5 & 5 & 5 \\
0 & 0 & $\mathbf{0}$ & 2 & 2 & 2 & 4 & 4 & 4 & 1 & 1 & $\mathbf{1}$ & 3 & 3 & 3 & 5 & 5 & 5 \\
1 & 1 & 1 & 3 & 3 & 3 & 5 & 5 & 5 & 2 & 2 & 2 & 4 & 4 & 4 & 0 & 0 & 0 \\
1 & 1 & 1 & 3 & 3 & 3 & 5 & 5 & 5 & 2 & 2 & 2 & 4 & 4 & 4 & 0 & 0 & 0 \\
\hline 2 & 2 & 2 & 4 & 4 & 4 & 0 & 0 & 0 & 3 & 3 & 3 & 5 & 5 & 5 & 1 & 1 & 1 \\
2 & 2 & 2 & 4 & 4 & 4 & 0 & 0 & 0 & 3 & 3 & 3 & 5 & 5 & 5 & 1 & 1 & 1 \\
3 & 3 & 3 & 5 & 5 & 5 & 1 & 1 & 1 & 4 & 4 & 4 & 0 & 0 & 0 & 2 & 2 & 2 \\
3 & 3 & 3 & 5 & 5 & 5 & 1 & 1 & 1 & 4 & 4 & 4 & 0 & 0 & 0 & 2 & 2 & 2 \\
\hline 4 & 4 & 4 & 0 & 0 & 0 & 2 & 2 & 2 & 5 & 5 & 5 & 1 & 1 & 1 & 3 & 3 & 3 \\
4 & 4 & 4 & 0 & 0 & 0 & 2 & 2 & 2 & 5 & 5 & 5 & 1 & 1 & 1 & 3 & 3 & 3 \\
5 & 5 & 5 & 1 & 1 & 1 & 3 & 3 & 3 & 0 & 0 & 0 & 2 & 2 & 2 & 4 & 4 & 4 \\
5 & 5 & 5 & 1 & 1 & 1 & 3 & 3 & 3 & 0 & 0 & 0 & 2 & 2 & 2 & 4 & 4 & 4 \\
\hline
\end{tabular}

A frequency rectangle of type $F R(12,18 ; 6)$ by Corollary 4

Before we prove Theorem 7 , we require the following number-theoretic observation.

Lemma 4. Let $b_{1}, b_{2}$ and $q$ be positive integers such that $q$ divides the product $b_{1} b_{2}$. Then there exist positive integers $q_{1}$ and $q_{2}$ such that $q_{1} q_{2}=q$ and $q_{1}$ divides $b_{2}$ and $q_{2}$ divides $b_{1}$.

Proof. Let $q=p_{0}^{s_{0}} p_{1}^{s_{1}} \ldots p_{m-1}^{s_{m-1}}$ be the prime factorization of $q$. Since $q$ divides $b_{1} b_{2}, b_{1}$ and $b_{2}$ must be of the form:

$$
\begin{aligned}
& b_{1}=B_{1} p_{0}^{\alpha_{0}} p_{1}^{\alpha_{1}} \ldots p_{m-1}^{\alpha_{m-1}}, \\
& b_{2}=B_{2} p_{0}^{\beta_{0}} p_{1}^{\beta_{1}} \ldots p_{m-1}^{\beta_{m-1}},
\end{aligned}
$$

where $p_{i}$ does not divide $B_{j}$ and $\alpha_{i}+\beta_{i} \geq s_{i}$ for all $i \in[m]$ and $j \in\{1,2\}$. Let

$$
q_{1}=p_{0}^{u_{0}} p_{1}^{u_{1}} \ldots p_{m-1}^{u_{m-1}}
$$

and

$$
q_{2}=p_{0}^{t_{0}} p_{1}^{t_{1}} \ldots p_{m-1}^{t_{m-1}}
$$

where $t_{i}:=\max \left\{0, s_{i}-\beta_{i}\right\}$ and $u_{i}=s_{i}-t_{i}$ for all $i \in[m]$.

Since $\alpha_{i}+\beta_{i} \geq s_{i}, t_{i} \leq \alpha_{i}$ for each $i \in[m]$, which implies that $q_{2}$ divides $b_{1}$. Also $s_{i}-\beta_{i} \leq t_{i}$ implies that $u_{i}=s_{i}-t_{i} \leq \beta_{i}$ and thus $q_{1}$ divides $b_{2}$. Finally observe that $q_{1} q_{2}=q$.

Theorem 7. Let $q$ be a divisor of $b_{1} b_{2}$. If there exist an array of type $I_{M+N}\left(q^{M}, q^{N} ; q\right)$, then there exists an array of type $I_{M+N+1}\left(q^{M} b_{1}, q^{N} b_{2} ; q\right)$.

Proof. By Lemma 4 we can choose $q_{1}, q_{2}$ such that $q_{1} q_{2}=q$ and $q_{1}$ divides $b_{2}$ and $q_{2}$ divides $b_{1}$.

Let $I^{\prime}$ be the array $I_{1}\left(q_{2}, q_{1} ; 1\right) \otimes I_{M+N}\left(q^{M}, q^{N} ; q\right)$ as shown in Table 2 


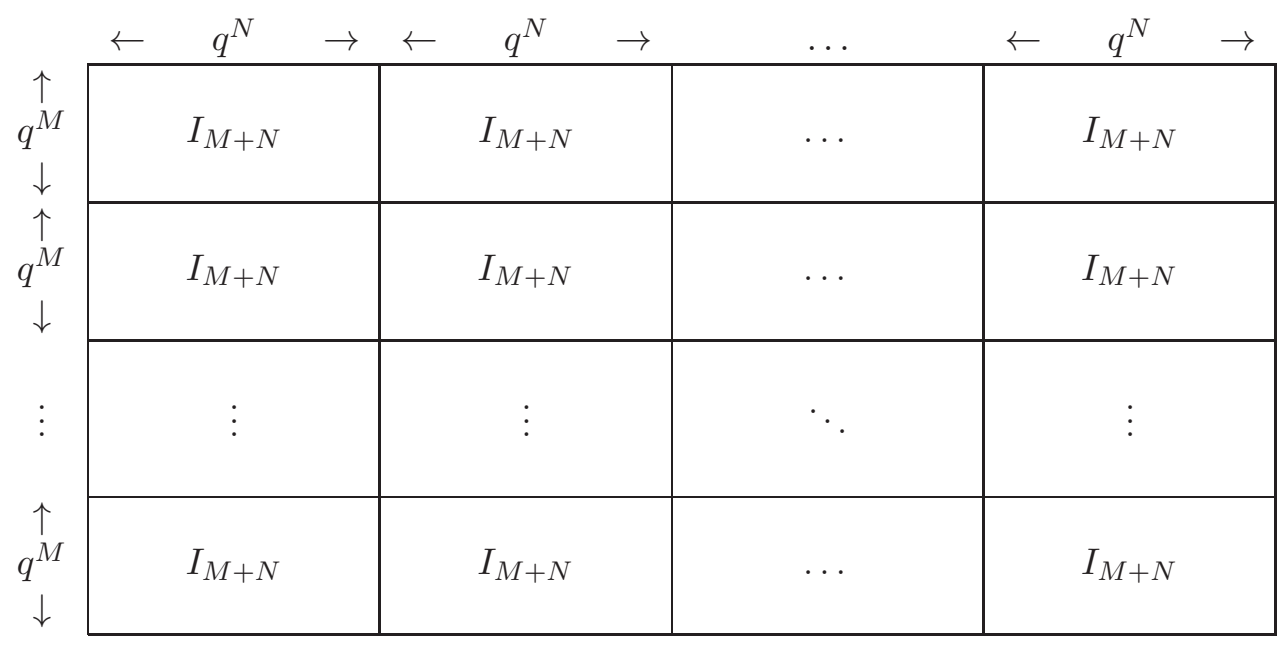

Table 2: $I^{\prime}=I_{1}\left(q_{2}, q_{1} ; 1\right) \otimes I_{M+N}\left(q^{M}, q^{N} ; q\right)$

Applying Corollary 4 with $\mu=q^{M-1} q_{2}$ and $\lambda=q^{N-1} q_{1}$, there exists a rectangular array $J^{\prime}$ of type $I_{1}\left(q^{M} q_{2}, q^{N} q_{1} ; q\right)$ such that for each $i \in\left[q^{M}\right]$ and $j \in\left[q^{N}\right]$, the set of cells

$$
\left\{\left(i^{\prime}, j^{\prime}\right) \mid i \equiv i^{\prime} \quad\left(\bmod q^{M}\right), j \equiv j^{\prime} \quad\left(\bmod q^{N}\right)\right\}
$$

contain each entry from $\left[q_{1} q_{2}\right]$ exactly once. It follows that the array $I^{\prime} \oplus J^{\prime}$ contains each sequence of length $M+N+1$ exactly once. Thus $I^{\prime} \oplus J^{\prime}$ is of type $I_{M+N+1}\left(q^{M} q_{2}, q^{N} q_{1} ; q\right)$. Finally, by Corollary $2, I_{1}\left(b_{1} / q_{2}, b_{2} / q_{1} ; 1\right) \otimes\left(I^{\prime} \oplus J^{\prime}\right)$ is an array of type $I_{M+N+1}\left(q^{M} b_{1}, q^{N} b_{2} ; q\right)$.

\section{The case $k=2$}

In this section we prove Theorem 2 in the case $k=2$. Given Theorem 4 it suffices to consider the existence of an array of type $I_{2}(m, n ; q)$ only in the case $q \in\{2,6\}$ and $m / q$ and $n / q$ odd. From Theorem 3 , arrays of type $I_{2}(2,2 ; 2)$ and $I_{2}(6,6 ; 6)$ do not exist. We next give another non-existence result.

Lemma 5. There does not exist an array of type $I_{2}(2, n ; 2)$ whenever $n / 2$ is odd.

Proof. Consider a frequency rectangle $F$ of type $F R(2, n ; 2)$. By permuting columns we can assume $F$ is in the following form:

\begin{tabular}{|llll|llll|}
\hline 0 & 0 & $\ldots$ & 0 & 1 & 1 & $\ldots$ & 1 \\
\hline 1 & 1 & $\ldots$ & 1 & 0 & 0 & $\ldots$ & 0 \\
\hline
\end{tabular}

Now consider any other frequency square $F^{\prime}$ of type $F R(2, n ; 2)$. Now since $n \equiv 2(\bmod 4)$, $F^{\prime}$ contains at least $\lfloor n / 4\rfloor+1$ symbols of the same type (say 0 ) in the first $n / 2$ cells of its first row. This implies there are at least $\lfloor n / 4\rfloor+11^{\prime} s$ in the second half of the first row and consequently we have $\lfloor n / 4\rfloor+10^{\prime} s$ in the second half of its second row. Thus if we 
superimpose $F$ and $F^{\prime}$, we get at least $2 \times\lfloor n / 4\rfloor+2>n / 2=2 n / 4$ ordered pairs of type $(0,0)$. Which shows $F$ and $F^{\prime}$ are not orthogonal.

Lemma 6. Let $m / 2$ and $n / 2$ be odd where $n \geq m>2$. Then there exists an array of type $I_{2}(m, n ; 2)$.

Proof. Let $m=2 l_{1}$ and $n=2 l_{2}$, where $l_{1}$ and $l_{2}$ are odd and $l_{2} \geq l_{1}>1$. Let $l_{2}=l_{1}+2 t$. Now by Theorem 3 there exists an array of type $I_{2}\left(2 l_{1}, 2 l_{1} ; 2\right)$. By Theorem 5 and Corollary 2 there exists an array of type $I_{2}\left(2 l_{1}, 4 t ; 2\right)$. Thus by Lemma 1, there exists an array of type $I_{2}\left(2 l_{1}, 2 l_{2} ; 2\right)$.

Next we consider when $q=6$. An array of type $I_{2}(6,12 ; 6)$ exists by Theorem 3 . We also exhibit an array of type $I_{2}(6,18 ; 6)$ :

$\begin{array}{llllllllllllllllll}13 & 24 & 35 & 40 & 51 & 02 & 15 & 24 & 30 & 43 & 51 & 02 & 10 & 24 & 33 & 45 & 51 & 02 \\ 34 & 43 & 01 & 52 & 20 & 15 & 34 & 45 & 01 & 52 & 23 & 10 & 34 & 40 & 01 & 52 & 25 & 13 \\ 43 & 32 & 10 & 25 & 04 & 53 & 41 & 32 & 13 & 20 & 04 & 55 & 41 & 32 & 15 & 23 & 04 & 50 \\ 22 & 11 & 54 & 03 & 45 & 30 & 22 & 11 & 54 & 05 & 40 & 33 & 22 & 11 & 54 & 00 & 43 & 35 \\ 50 & 05 & 23 & 31 & 12 & 44 & 53 & 00 & 25 & 31 & 12 & 44 & 55 & 03 & 20 & 31 & 12 & 44 \\ 04 & 50 & 42 & 14 & 33 & 21 & 00 & 53 & 42 & 14 & 35 & 21 & 03 & 55 & 42 & 14 & 30 & 21\end{array}$

An array of type $I_{2}(6,18 ; 6)$.

By Lemma 1, we thus obtain the following.

Lemma 7 . There exists an array of type $I_{2}\left(6 l_{1}, 6 l_{2} ; 6\right)$ if and only if $\left(l_{1}, l_{2}\right) \neq(1,1)$.

\section{The case $k \geq 3$.}

It now suffices to prove the case $k \geq 3$ in order to prove Theorem 2 .

Theorem 8. Let $k \geq 3, q|m, q| n$ and $q^{k} \mid m n$. Then there exist an array of type $I_{k}(m, n ; q)$.

Proof. Trivially, if an array of type $I_{k}(m, n ; q)$ exists, then an array of type $I_{\ell}(m, n ; q)$ exists for each $1 \leq \ell<k$. Thus we may assume that $k=\max \left\{t: q^{t} \mid m n\right\}$. Let $m n=q^{k} b$.

Consider the prime factorization of $q$ :

$$
q=p_{0}^{s_{0}} p_{1}^{s_{1}} \ldots p_{l-1}^{s_{l-1}} .
$$

For each $r \in[l]$, let $i_{r}=\max \left\{t: q_{r}^{t} \mid m\right\}$ and $j_{r}=\max \left\{t: q_{r}^{t} \mid n\right\}$, where $q_{r}=p_{r}^{s_{r}}$. Thus $m$ and $n$ can be expressed as:

$$
m=q_{0}^{i_{0}} \ldots q_{l-1}^{i_{l-1}} p_{0}^{\alpha_{0}} \ldots p_{l-1}^{\alpha_{l-1}} a_{1}, \quad n=q_{1}^{j_{1}} \ldots q_{l}^{j_{l}} p_{0}^{\beta_{0}} \ldots p_{l-1}^{\beta_{l-1}} a_{2}
$$

with $\alpha_{r}, \beta_{r}<s_{r}$ and $p_{r} \nmid a_{1}$ and $p_{r} \nmid a_{2}$ for each $r \in[l]$. Now for any $c \in[l]$ we have the following two cases: 
Case I: When $\alpha_{c}+\beta_{c}<s_{c}$.

In this case, $i_{c}+j_{c}$ is the largest power of $q_{c}$ which divides $m n$, and thus $i_{c}+j_{c}$ is the largest power of $q_{c}$ which divides $k$. By Theorem 5 and Corollary 3 if $\left(i_{c}, j_{c}, q_{c}\right) \neq(1,1,2)$, there exists an array of type $I_{k}\left(q_{c}^{i_{c}} p_{c}^{\alpha_{c}}, q_{c}^{j_{c}} p_{c}^{\beta_{c}} ; q_{c}\right)$, where $k=i_{c}+j_{c}$. However if $\left(i_{c}, j_{c}, q_{c}\right)=$ $(1,1,2)$, then $s_{c}=1, \alpha_{c}=\beta_{c}=0$ and $2^{3}$ does not divide $m n$, contradicting $k \geq 3$.

Case II: When $\alpha_{c}+\beta_{c} \geq s_{c}$.

Since $\alpha_{c}, \beta_{c}<s_{c}$, this implies $\alpha_{c}+\beta_{c}<2 s_{c}$ and therefore $i_{c}+j_{c}+1$ is the largest power of $q_{c}$ which divides $k$. By combining Theorem 5 and Theorem 7 we obtain an array of type $I_{k}\left(q_{c}^{i_{c}} p_{c}^{\alpha_{c}}, q_{c}^{j_{c}} p_{c}^{\beta_{c}} ; q_{c}\right)$ where $k=i_{c}+j_{c}+1$.

Thus in both cases for each $c \in[l]$ we obtain an array of type $I_{k}\left(q_{c}^{i_{c}} p_{c}^{\alpha_{c}}, q_{c}^{j_{c}} p_{c}^{\beta_{c}} ; q_{c}\right)$ and by taking their Kronecker product (see Corollary 1), we can construct an array of type $I_{k}\left(\frac{m}{a_{1}}, \frac{n}{a_{2}} ; q\right)$ where $a_{1}$ and $a_{2}$ are defined in equation (44). Finally, by applying Corollary 2 we obtain an array of type $I_{k}(m, n ; q)$, which completes the proof.

The previous section and Theorem 8 together imply Theorem 2 .

\section{References}

[1] R. Bailey. Patterns of confounding in factorial designs. Biometrika, 64(3):597-603, 1977.

[2] R. Bailey. Factorial design and abelian groups. Linear Algebra and its Applications, 70:349-368, 1985.

[3] C. J. Brien, R. Bailey, T. T. Tran, J. Boland, et al. Quasi-latin designs. Electronic Journal of Statistics, 6:1900-1925, 2012.

[4] T. Britz, N. Cavenagh, A. Mammoliti, and I. Wanless. Mutually orthogonal binary frequency squares. The Electronic Journal of Combinatorics, 27(3):P3.7, 2020.

[5] C.-S. Cheng and P.-W. Tsai. Templates for design key construction. Statistica Sinica, pages 1419-1436, 2013.

[6] K. C. Choi and S. Gupta. Confounded row-column designs. Journal of statistical planning and inference, 138(1):196-202, 2008.

[7] S. Dash, R. Parsad, and V. Gupta. Row-column designs for $2^{n}$ factorial 2-colour microarray experiments for estimation of main effects and two-factor interactions with orthogonal parameterization. Agricultural research, 2(2):172-182, 2013.

[8] A. Datta, S. Jaggi, E. Varghese, and C. Varghese. Generalized confounded row-column designs. Communications in Statistics - Theory and Methods, 46(12):6213-6221, 2017.

[9] A. Dean and S. Lewis. A unified theory for generalized cyclic designs. Journal of Statistical Planning and Inference, 4(1):13-23, 1980.

[10] A. Dean and S. Lewis. Multidimensional designs for two-factor experiments. Journal of the American Statistical Association, 87(420):1158-1165, 1992. 
[11] W. Federer, A. Hedayat, and J. Mandeli. Pairwise orthogonal f-rectangle designs. Journal of statistical planning and inference, 10(3):365-374, 1984.

[12] W. T. Federer et al. On the existence and construction of a complete set of orthogonal $f(4 t ; 2 t, 2 t)$-squares design. The Annals of Statistics, 5(3):561-564, 1977.

[13] J. Godolphin. Construction of row-column factorial designs. Journal of the Royal Statistical Society: Series B (Statistical Methodology), 81(2):335-360, 2019.

[14] A. Hedayat, D. Raghavarao, E. Seiden, et al. Further contributions to the theory of $f$-squares design. The Annals of Statistics, 3(3):712-716, 1975.

[15] J. John and S. Lewis. Factorial experiments in generalized cyclic row-column designs. Journal of the Royal Statistical Society: Series B (Methodological), 45(2):245-251, 1983.

[16] A. Kobilinsky. Confounding in relation to duality of finite abelian groups. Linear algebra and its applications, 70:321-347, 1985.

[17] C. Laywine and G. Mullen. Discrete Mathematics Using Latin Squares. 1484 Series. Wiley, 1998.

[18] C. F. Laywine and G. L. Mullen. A table of lower bounds for the number of mutually orthogonal frequency squares. Ars Combinatoria, 59:85-96, 2001.

[19] M. Li, Y. Zhang, and B. Du. Some new results on mutually orthogonal frequency squares. Discrete Mathematics, 331:175-187, 2014.

[20] V. C. Mavron. Frequency squares and affine designs. The Electronic Journal of Combinatorics, 7(1):56, 2000.

[21] C. R. Rao. Confounded factorial designs in quasi-latin squares. Sankhyā: The Indian Journal of Statistics, pages 295-304, 1946.

[22] D. Street. Generalized hadamard matrices, orthogonal arrays and f-squares. Ars Combinatoria, 8:131-141, 1979.

[23] P. Wang. Orthogonal main-effect plans in row-column designs for two-level factorial experiments. Communications in Statistics-Theory and Methods, 46(21):10685-10691, 2017.

[24] E. Williams and J. John. Row-column factorial designs for use in agricultural field trials. Journal of the Royal Statistical Society: Series C (Applied Statistics), 45(1):3946, 1996.

[25] F. Yates. The design and analysis of factorial experiments. Imperial Bureau of Soil Science Harpenden, UK, 1978. 